Draft VERSION MAY 11, 2021

Typeset using $\mathrm{LAT}_{\mathrm{E}} \mathrm{X}$ twocolumn style in AASTeX62

\title{
The IceCube Pie Chart: Relative Source Contributions to the Cosmic Neutrino Flux
}

\author{
I. Bartos, ${ }^{1, *}$ D. Veske, ${ }^{2}$ M. Kowalski,${ }^{3,4,5}$ Z. Márka, ${ }^{5}$ And S. MÁrka ${ }^{2}$ \\ ${ }^{1}$ Department of Physics, University of Florida, PO Box 118440, Gainesville, FL 32611-8440, USA \\ ${ }^{2}$ Department of Physics, Columbia University, New York, NY 10027, USA \\ ${ }^{3}$ Deutsches Elektronen Synchrotron DESY, Platanenallee 6, 15738 Zeuthen, Germany \\ ${ }^{4}$ Institut fur Physik, Humboldt-Universit at zu Berlin, D-12489 Berlin, Germany \\ ${ }^{5}$ Columbia Astrophysics Laboratory, Columbia University, New York, NY 10027, USA
}

\begin{abstract}
Neutrino events from IceCube have recently been associated with multiple astrophysical sources. Interestingly, these likely detections represent three distinct astrophysical source types: active galactic nuclei (AGN), blazars, and tidal disruption events (TDE). Here we compute the expected contributions of AGNs, blazars and TDEs to the overall cosmic neutrino flux detected by IceCube based on the associated events, IceCube's sensitivity, and the source types' astrophysical properties. We find that, despite being the most commonly identified sources, blazars cannot contribute more than $11 \%$ of the total flux (90\% credible level), consistent with existing limits from stacked searches. On the other hand, either AGNs or TDEs could contribute more than $50 \%$ of the total flux (90\% credible level). We also find that so-far unknown source types contribute at least $10 \%$ of the total cosmic flux with a probability of $80 \%$. We assemble a pie chart that shows the most likely fractional contribution of each source type to IceCube's total neutrino flux.
\end{abstract}

\section{INTRODUCTION}

The Universe produces a quasi-diffuse flux of highenergy $(>\mathrm{TeV}$ ) neutrinos (hereafter cosmic neutrino flux; Aartsen et al. 2013; Aartsen et al. 2014), whose properties are now well characterized (Aartsen et al. 2016). The origin of this cosmic flux is, nevertheless, not yet understood.

High-energy neutrinos have recently been identified from several distinct astrophysical sites. These sources include the TXS 0506+056 blazar (Aartsen et al. 2018a,b), a nearby Seyfert galaxy (NGC 1068; Aartsen et al. 2020a), and a tidal disruption event (TDE; AT2019dsg; Stein et al. 2020). Several other blazars have also been identified with probable high-energy neutrino associations (Kadler et al. 2016; Kun et al. 2021). At the same time, the contribution of some other source types to the overall flux, such as gamma-ray bursts (GRBs) and blazars, has been significantly constrained (Abbasi et al. 2012; Ackermann et al. 2016; Yuan et al. 2020).

In this paper we evaluate the expected overall contribution of different source types to the cosmic neutrino flux based on the identified individual neutrino sources. We show that the widely different properties of differ-

*imrebartos@ufl.edu ent source types mean that the same number of detections translates to different expected contributions to the overall flux. In addition, computing the expected contribution of each source type to the overall cosmic neutrino flux allows us to estimate the fraction of the overall flux that arrives from so far unidentified source types, i.e sources that are not AGNs, blazars or TDEs.

An additional goal of the present description is to demonstrate how different source features, such as their number density, number of detections or their cosmic rate evolution, contributes to their estimated contribution to the overall neutrino flux. Therefore, before discussing our Bayesian estimate that includes most accessible details about the source populations, we first compute fractional contributions through simpler estimates in which the role of different source properties is more accessible.

The paper is organized as follows. We first carry out our simplest "warm-up" calculation of fractional contributions in Section 2 using detections that followed high-energy neutrino alerts publicly released by IceCube. Next, we introduce a simplified model in Section 3 that highlights the relative importance of population properties. We then introduce our most detailed and realistic method in Section 4 and its implementation in 5 to obtain the estimated relative contributions for both detected and unknown source types. Results for this 
most detailed model are presented in Section 6. We summarize our conclusions in Section 7.

\section{WARMING UP: THE FRACTION OF DISCOVERIES}

It is instructive to evaluate the fraction of high-energy neutrino alerts (Aartsen et al. 2017a; Blaufuss et al. 2019) that lead to likely associations with counterparts. If one further factors in the completness of the catalog of potential counterparts, one can translate this fraction into a constraint on the contribution of the specific source class to the total neutrino flux. Below we examine blazars and TDEs which were identified so far in association with IceCube's neutrino alerts.

We first consider blazars that were found in association with the $N_{\text {alert }} \sim 60$ alerts $^{12}$ sent to the community by IceCube so far: besides the well known TXS0506+056 (Aartsen et al. 2018b), more recently PKS 1502+106 - another exceptionally bright blazarwas identified in coincident with a well localized neutrino track (Taboada \& Stein (2019)). Other claimed associations are less significant (e.g. GB6 J1040+0617, Garrappa et al. (2019)) and/or have not been found in associations with alerts (PKS 1424-41; Kadler et al. 2016), and since we do not consider backgrounds here, they are not counted in this simple exercise. We hence count two detections, $N_{\text {det }}=2$. We take into account that roughly half of IceCube's alerts are estimated to be of astrophysical origin ( signalness $^{3} s \approx 0.5$ ). In addition, based on their gamma-ray flux, we assume that a fraction $f \sim 0.7$ of the high-energy neutrino flux from this source type are from electromagnetically resolved blazars. With these quantities we can estimate the fraction of cosmic, high-energy neutrinos due to a blazar population: $N_{\text {det }} / N_{\text {alert }} / f / s=0.10$.

Likewise for TDEs, Stein et al. (2020) associated one TDE $\left(N_{\text {det }}=1\right)$ from a search of alerts $\left(N_{\text {alerts }}=9\right)$, with an estimated completeness factor $f=0.4$. These numbers translate to a fraction of the diffuse neutrino flux due to a population of TDEs of $N_{\text {det }} / N_{\text {alert }} / \mathrm{f} / \mathrm{s}=$ 0.55 .

These initial estimates come with significant caveats, e.g. they do not take into account the significance of the observations, and moreover are not generalizable to

\footnotetext{
1 https://gcn.gsfc.nasa.gov/amon_hese_events.html.

2 https://gcn.gsfc.nasa.gov/amon_ehe_events.html.

3 IceCube neutrino alerts have an assigned quantity called signalness, defined as the probability that the neutrino signal came from an astrophysical, as opposed to an atmospheric source, based on the neutrino energy, sky location and time of arrival, but without accounting for information from electromagnetic observations Blaufuss et al. (2019).
}

observations that are not based on single-neutrino coincidences. Nevertheless, they are illustrative of a key point, namely the importance of the completeness of the catalog of counterparts in extrapolating the flux to a full population of sources.

\section{SIMPLE MODEL}

In order to characterize the role of different population properties in connecting detections with the estimated contribution to the overall cosmic neutrino flux, we consider the following simple model. We separately consider continuous neutrino sources that are detected through time-integrated searches, and searches that identify sources via the detection of a single highenergy neutrino.

\subsection{Time-integrated detection}

Time-integrated searches are particularly relevant for continuous sources with high number densities where the detection of multiple neutrinos is necessary to claim detection. Let $F_{\nu, 0}$ be the neutrino threshold above which such a time-integrated search is able to identify a neutrino flux. For simplicity we neglect any dependence on the sources' neutrino spectrum and sky location.

Let sources within a given source type be uniformly distributed in the local universe with number density $\rho$. Let each source have unknown identical neutrino luminosity $L_{\nu}$. The maximum distance $r_{\max }$ within which these sources can be detected is

$$
r_{\max }=\sqrt{\frac{L_{\nu}}{4 \pi F_{\nu, 0}}}
$$

Within this distance the expected number of sources is

$$
\left\langle N_{\text {det }}\right\rangle=\frac{4}{3} \pi r_{\max }^{3} \rho
$$

We will consider the number $N_{\text {det }}$ of detected sources to be the best estimate of the expected number of detections, i.e. $\left\langle N_{\text {det }}\right\rangle=N_{\text {det }}$. With this, the expected neutrino flux from the considered source type within $r_{\max }$ is

$$
F_{\nu, \mathrm{r}}=\int_{0}^{r_{\max }} d r 4 \pi r^{2} \rho \frac{L_{\nu}}{4 \pi r^{2}}=\rho L_{\nu} r_{\max }=3 F_{\nu, 0} N_{\mathrm{det}}
$$

where we made use of Eqs. 1 and 2 to change variables.

Let $f_{\mathrm{r}}\left(r_{\max }\right)$ be the fraction of the total neutrino flux from a given source type that comes from sources within distance $r_{\max }$. For not too large distances we approximate $f_{\mathrm{r}}\left(r_{\max }\right)=f_{0} r_{\max }$, where $f_{0}$ is a source-typedependent constant (Bartos et al. 2017). With this, we can express the overall flux expected from the considered 
source type as

$$
F_{\nu, \text { tot }}^{\text {(int })}=\frac{F_{\nu, \mathrm{r}}\left(r_{\max }\right)}{f_{\mathrm{r}}\left(r_{\max }\right)}=\frac{3 F_{\nu, 0} N_{\mathrm{det}}}{f_{0} r_{\max }} \approx 5 F_{\nu, 0} f_{0}^{-1} N_{\text {det }}^{2 / 3} \rho^{1 / 3}
$$

where the superscript "(int)" refers to time-integrated detection. We see that, for the same number of detections, the corresponding flux can be very different due to its dependence on $\rho$, which can vary orders of magnitude between sources.

\subsection{Single-neutrino detection}

For rare and/or transient sources, a single high-energy neutrino can be sufficient to claim detection. Let $F_{\nu, 1}$ be the source flux for which the expected number of detected neutrinos is 1 during the observing period $T_{\text {obs }}$. For transient sources with duration $\tau$ the corresponding actual source flux is $T_{\mathrm{obs}} / \tau$ times higher.

Let sources within a given source type be uniformly distributed in the local universe with number density $\rho$. Let each source have identical neutrino luminosity $L_{\nu}$. For transient sources, we will adopt the same notation defining $\rho=\mathcal{R} T_{\text {obs }}$ with source rate density $\mathcal{R}$, and $L_{\nu}=E_{\nu} T_{\text {obs }}^{-1}$ with $E_{\nu}$ total energy radiated in highenergy neutrinos.

A single neutrino can only lead to discovery if it is directionally (and temporally for transients) coincident with a source candidate. A single neutrino can be detected with non-vanishing probability even from distant sources. Therefore, single-neutrino searches will not be limited by a distance threshold for detection as in the time-integrated case. Instead, a solution is that the number of source candidates considered in the search is capped in order to limit the search's trial factor. Source candidates will be selected for a search such that those expected to have the highest neutrino flux at Earth are included. If all sources produce the same neutrino flux then the closest sources are selected. If we limit the source candidates in a search to the number $N_{\max }$, then this corresponds to an effective maximum search distance of

$$
r_{\max }=\left(\frac{3 N_{\max }}{4 \pi \rho}\right)^{1 / 3}
$$

The expected number of detected sources within this distance is

$$
\left\langle N_{\mathrm{det}}\right\rangle=\int_{0}^{r_{\max }} d r 4 \pi r^{2} \rho \frac{L_{\nu}}{4 \pi r^{2}} F_{\nu, 1}^{-1}=\rho L_{\nu} r_{\max } F_{\nu, 1}^{-1}
$$

where we assumed that the expected number of detected neutrinos from a single source is $\ll 1$.

We will consider the number $N_{\text {det }}$ of detected sources to be the best estimate of the expected number of detec- tions, i.e. $\left\langle N_{\text {det }}\right\rangle=N_{\text {det }}$. With this, the expected neutrino flux from the considered source type within $r_{\max }$ is

$F_{\nu, \mathrm{r}}\left(r_{\max }\right)=\int_{0}^{r_{\max }} d r 4 \pi r^{2} \rho \frac{L_{\nu}}{4 \pi r^{2}}=\rho L_{\nu} r_{\max }=F_{\nu, 1} N_{\mathrm{det}}$,

where we made use of Eq. 6 to change variables.

Similarly to the previous section, we introduce the fraction $f_{\mathrm{r}}\left(r_{\max }\right)=f_{0} r_{\max }$ of the total neutrino flux from a given source type that comes from sources within distance $r_{\max }$. With this, we can express the overall flux expected from the considered source type as

$$
F_{\nu, \text { tot }}^{(\text {single })}=\frac{F_{\nu, \mathrm{r}}\left(r_{\max }\right)}{f_{\mathrm{r}}\left(r_{\max }\right)}=\frac{F_{\nu, 1} N_{\mathrm{det}}}{f_{0} r_{\max }} \approx \frac{1.6 F_{\nu, 1} N_{\mathrm{det}} \rho^{1 / 3}}{f_{0} N_{\max }^{1 / 3}}
$$

where the superscript "(single)" refers to single-neutrino detection. This formula is very similar to the one we obtained for time integrated searches (see Eq. 4), and shows that the number density (or rate density) of sources can substantially alter the expected contribution from a source type.

\subsection{Estimated contribution based on simple model}

We now estimate the contribution of different detected source types to the cosmic neutrino flux. We additionally consider sources that have so far been undetected to gauge how strong constraint their non-detection represents to their contribution to the overall flux.

\subsection{Flux fraction}

First, we computed $f_{0}$ for different source populations, which is needed to estimate their total flux. We considered (non-jetted) AGNs, tidal disruption events (TDEs) and, for comparison, populations that follow the cosmic star-formation rate. Our results are shown in Fig. 1. For AGNs we adopted their number density for bolometric luminosity $>10^{43} \mathrm{erg} \mathrm{s}^{-1}$ from Lacy et al. (2015). For TDEs we adopted their cosmic density evolution from Sun et al. (2015), while we adopted the cosmic star formation rate from Li (2008).

\subsubsection{Blazars}

We used $N_{\text {det }}=3, \rho_{\text {blazar }}=10^{-9} \mathrm{Mpc}^{-3}$ and $T_{\text {obs }}=$ $5 \mathrm{yr}$. We considered blazars to be detectable with single-neutrino searches. We adopted $\mathcal{F}_{\nu, 1}=4 \times$ $10^{-7} \mathrm{erg} \mathrm{cm}^{-2} \mathrm{~s}^{-1}\left(1 \mathrm{yr} / T_{\mathrm{obs}}\right)$, where we assumed a neutrino spectral index of 3 and neutrino energy range $\varepsilon_{\nu} \in[100 \mathrm{GeV}, 100 \mathrm{PeV}] .{ }^{4}$ We further adopted $f_{0}=$

\footnotetext{
${ }^{4}$ Here we used the flux corresponding to an expected number of 1 detected neutrino in the $\varepsilon_{\nu} \in[100 \mathrm{TeV}, 100 \mathrm{PeV}]$ from IceCube
} 


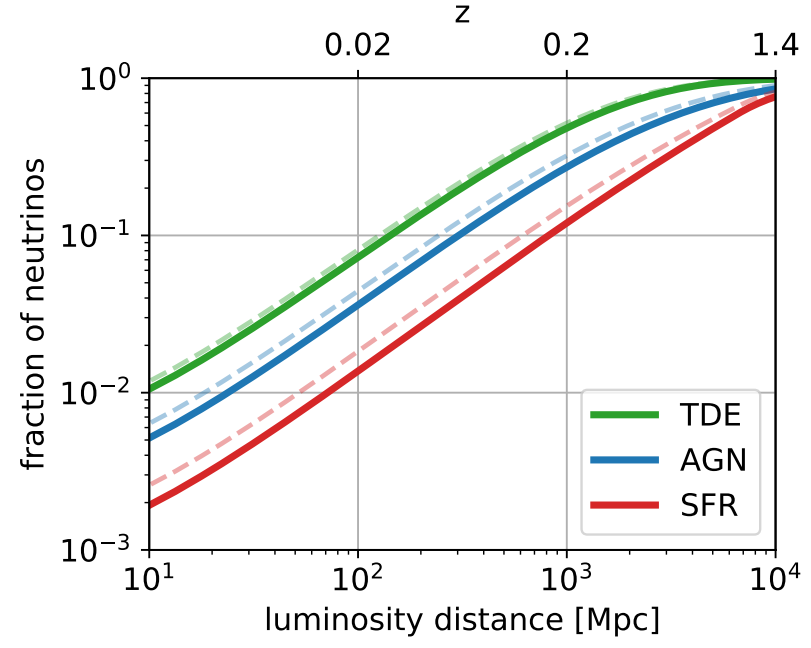

Figure 1. Fraction of detected high-energy neutrinos in IceCube expected from sources closer than luminosity distance $d_{\mathrm{L}}$ as a function of $d_{\mathrm{L}}$. Shown are results for AGNs/blazars, TDEs and source populations that track the cosmic star formation rate (SFR; see legend). Solid lines show results for assumed neutrino spectral density $d N / d E \propto E^{-2.5}$. For comparison we show for $d N / d E \propto E^{-3}$ (dashed lines).

$5 \times 10^{-4} \mathrm{Mpc}^{-1}$ (see Fig. 1, we considered the same fraction for AGNs and blazars) and $N_{\max }=100$ (see Aartsen et al. (2020a)).

With these parameters, we obtained $F_{\nu, \text { tot }}^{(\text {blazar })}=8 \times$ $10^{-8} \mathrm{erg} \mathrm{cm}^{-2} \mathrm{~s}^{-1}$.

\subsubsection{AGNs}

For AGNs we used $N_{\text {det }}=1$ and $\rho_{\text {AGN }}=10^{-4} \mathrm{Mpc}^{-3}$. We considered AGNs to be detectable with a timeintegrated search. We adopted $F_{\nu, 0}=2 \times 10^{-10} \mathrm{erg} \mathrm{cm}^{-2} \mathrm{~s}^{-1}$ where we assumed a neutrino spectral index of 3 and energy range $\varepsilon_{\nu} \in[100 \mathrm{GeV}, 100 \mathrm{PeV}]$ (see Aartsen et al. (2020a)). We further adopted $f_{0}=5 \times 10^{-4} \mathrm{Mpc}^{-1}$.

With these parameters, we obtained $F_{\nu, \text { tot }}^{(\mathrm{AGN})}=9 \times$ $10^{-8} \mathrm{erg} \mathrm{cm}^{-2} \mathrm{~s}^{-1}$.

\subsubsection{TDEs}

For TDEs we used $N_{\text {det }}=1$ and $\rho_{\mathrm{TDE}}=\mathcal{R}_{\mathrm{TDE}} T_{\mathrm{obs}}=$ $10^{-9.5} \mathrm{Mpc}^{-3}$ where we assumed $T_{\mathrm{obs}}=5 \mathrm{yr}$. We considered TDEs to be detectable with single-neutrino searches. We adopted $\mathcal{F}_{\nu, 1}=4 \times 10^{-7} \mathrm{erg} \mathrm{cm}^{-2} \mathrm{~s}^{-1}\left(1 \mathrm{yr} / T_{\text {obs }}\right)$, assuming a neutrino spectral index of 3 and neutrino energy range $\varepsilon_{\nu} \in[100 \mathrm{GeV}, 100 \mathrm{PeV}]$. We adopted $f_{0}=9 \times 10^{-4} \mathrm{Mpc}^{-1}$.

Collaboration (2016). We then extrapolated this flux to the energy range $\varepsilon_{\nu} \in[100 \mathrm{GeV}, 100 \mathrm{PeV}]$ to make it compatible with the flux from the time-integrated search. We adopted a neutrino spectral index of 3 for the extrapolation.
In order to determine $N_{\max }$, we set the maximum detectable distance of TDEs to be $r_{\max }$,TDE $=1 \mathrm{Gpc}$ given the distance range of past identified TDEs (van Velzen et al. 2020). We assumed that electromagnetic follow-up of $\gtrsim 100 \mathrm{TeV}$ neutrinos could identify every TDE within $r_{\max , \mathrm{TDE}}$. We set $N_{\max }=4 / 3 \pi r_{\max , \mathrm{TDE}}^{3} \rho_{\mathrm{TDE}} \approx 1$.

With these parameters, we obtained $F_{\nu, \text { tot }}^{(\mathrm{TDE})}=5 \times$ $10^{-8} \mathrm{erg} \mathrm{cm}^{-2} \mathrm{~s}^{-1}$.

\subsubsection{Gamma-ray bursts}

While no significant neutrino emission has been associated with gamma-ray bursts (GRBs), here we considered the corresponding total flux from GRBs assuming $N_{\text {det }}=2.3$, which is the $90 \%$ confidence level upper limit corresponding to no detections. This can give us a picture of the total flux contribution that is inconsistent with the lack of association. We adopted a local density of $\rho_{\mathrm{GRB}}=\mathcal{R}_{\mathrm{GRB}} T_{\mathrm{obs}}=10^{-8} \mathrm{Mpc}^{-3}$ where we assumed $T_{\text {obs }}=10 \mathrm{yr}$ (Wanderman \& Piran 2010). We considered GRBs to be detectable with single-neutrino searches.

Given the short duration and rarity of GRBs, and their easy observability with all-sky gamma-ray detectors, single-neutrino searches can consider neutrinos below the $100 \mathrm{TeV}$ limit used for other single-neutrino searches. Adopting a threshold of $1 \mathrm{TeV}$ and a neutrino spectral index of 3 , we consider a threshold flux $\mathcal{F}_{\nu, 1}=4 \times 10^{-9} \mathrm{erg} \mathrm{cm}^{-2} \mathrm{~s}^{-1}\left(1 \mathrm{yr} / T_{\text {obs }}\right)$, i.e. 100 times lower than for an energy threshold of $100 \mathrm{TeV}$.

We adopted $f_{0}=2 \times 10^{-4} \mathrm{Mpc}^{-1}$, similar to the fraction expected for a population tracing the star formation rate. We took $N_{\max }=1000$, comparable to the total number of detected GRBs.

With these parameters, we obtained $F_{\nu \text {,tot }}^{(\mathrm{GRB})} \lesssim$ $10^{-9} \mathrm{erg} \mathrm{cm}^{-2} \mathrm{~s}^{-1}$. We conclude that the lack of detection presents a strong constraint on the GRB contribution to the total cosmic neutrino flux, their contribution is $<1 \%$. This limit is consistent with constraints from searches by IceCube for neutrinos coincident with GRBs (Aartsen et al. 2017b).

\subsubsection{Core-collapse supernovae}

No significant neutrino emission has been associated with supernovae. Here we considered the corresponding total flux from core-collapse supernovae assuming $N_{\text {det }}=2.3$ to characterize the limit this lack of detection represents.

We adopted a core-collapse supernova rate density of $\sim 10^{-4} \mathrm{Mpc}^{-3} \mathrm{yr}^{-1}$ Taylor et al. (2014), and assumed that neutrino emission is significant for a duration of $T_{\text {obs }}=10 \mathrm{yr}$. With this we obtain an effective density $\rho_{\mathrm{SN}}=10^{-3} \mathrm{Mpc}^{-3}$. We considered supernovae to be detectable with time-integrated searches. 
We adopted $F_{\nu, 0}=2 \times 10^{-10} \mathrm{erg} \mathrm{cm}^{-2} \mathrm{~s}^{-1}$ where we assumed a neutrino spectral index of 3 and energy range $\varepsilon_{\nu} \in[100 \mathrm{GeV}, 100 \mathrm{PeV}]$ (see Aartsen et al. 2020a). We further adopted $f_{0}=2 \times 10^{-4} \mathrm{Mpc}^{-1}$ assuming that the supernova rate traces the star formation rate.

With these parameters, we obtained $F_{\nu, \text { tot }}^{(\mathrm{CCSN})} \lesssim$ $10^{-6} \mathrm{erg} \mathrm{cm}^{-2} \mathrm{~s}^{-1}$, i.e. greater than IceCube's overall flux. We therefore conclude that the lack of observation does not present a meaningful constraint on the contribution of supernovae to the total cosmic neutrino flux.

\section{FULL BAYESIAN MODEL}

We now turn to a more detailed derivation of the expected neutrino flux from different source populations where we take into account the cosmic evolution of sources and the statistical uncertainty of the number of detections.

\subsection{Probability density of the expected number of detections}

Assume we have a set of $N_{\text {tot }}$ detection candidates for source type $S$. Each candidate is either from the astrophysical source of interest or from the background. For our purposes here, astrophysical neutrinos from unassociated sources are counted as background. Candidate $i$ has a set of reconstructed parameters denoted with $\vec{x}_{i}$. The set of reconstructed parameters for all candidates is denoted with $\vec{x}=\left\{\vec{x}_{1}, \vec{x}_{2} \ldots \vec{x}_{\mathrm{N}_{\text {tot }}}\right\}$.

Let $p\left(\vec{x}_{i} \mid S\right)$ and $p\left(\vec{x}_{i} \mid B\right)$ be the probability densities of observing $\vec{x}_{i}$ from an astrophysical source of type $S$ and from the background, respectively. We compute the probability density of the expected number of detected events, denoted with $N_{\text {det }}$ as (Farr et al. 2015) (note the similarity to Eq. 7 in Braun et al. 2008):

$$
\begin{aligned}
p\left(N_{\mathrm{det}} \mid \vec{x}\right) \propto \int & d N_{\mathrm{B}} \prod_{i=1}^{N_{\text {tot }}}\left[N_{\mathrm{det}} p\left(\vec{x}_{i} \mid S\right)+N_{\mathrm{B}} p\left(\vec{x}_{i} \mid B\right)\right] \\
& \times e^{-\left(N_{\mathrm{det}}+N_{\mathrm{B}}\right)} \frac{\pi\left(N_{\mathrm{det}}\right)}{\sqrt{N_{\mathrm{B}}}}
\end{aligned}
$$

where $N_{\mathrm{B}}$ is the expected number of detected background events, which we marginalize over. We used the Poisson Jeffreys prior for $N_{\mathrm{B}}$. We define the prior probability density $\pi\left(N_{\text {det }}\right)$ of $N_{\text {det }}$ implicitly by assuming that the prior probability density of the total flux from a given source type is uniformly distributed between 0 and IceCube's measured cosmic neutrino flux minus the flux of the other sources. Therefore, we will have a three-dimensional prior probability density for the three sources considered below, with uniform probability density and with the boundary condition that the sum of the total flux from the three sources cannot exceed IceCube's measured flux.

\subsection{Computing the neutrino luminosity of individual sources}

If we know the expected number of detected sources, we can compute the related neutrino luminosity of individual sources. This computation, however, also depends on the luminosity's probability density.

Here we will assume that the neutrino luminosity $L_{\nu}$ of a source depends on its electromagnetic luminosity $L_{\gamma}$. For simplicity, we will assume that $L_{\nu}=\alpha_{\gamma \nu} L_{\gamma}$ with unknown $\alpha_{\gamma \nu}$ constant. We further assume that we know the number density $\rho\left(z, L_{\gamma}\right)$ of a continuous source type as a function of redshift $z$ and $L_{\gamma}$.

With this, we can compute the expected number of detections:

$$
N_{\mathrm{S}, \text { cont. }}=\int d z d L_{\nu} \frac{4 \pi c}{H(z)} \frac{d_{\mathrm{L}}(z)^{2}}{(1+z)^{2}} \rho\left(z, \frac{L_{\nu}}{\alpha_{\gamma \nu}}\right) p_{\operatorname{det}}\left(z, L_{\nu}\right) .
$$

Here, $d_{\mathrm{L}}$ is the luminosity distance and $p_{\text {det }}\left(z, L_{\nu}\right)$ is the probability that a source with luminosity $L_{\nu}$ at redshift $z$ will be detected. We can compute the expected detection rate for transient sources as well, where we also need to take into account time dilation, obtaining

$$
\begin{aligned}
N_{\mathrm{S}, \text { trans. }}=T_{\mathrm{obs}} & \int d z d E_{\nu} \frac{4 \pi c}{H(z)} \frac{d_{\mathrm{L}}(z)^{2}}{(1+z)^{3}} \\
& \times \mathcal{R}\left(z, \frac{E_{\nu}}{\alpha_{\gamma \nu}}\right) p_{\operatorname{det}}\left(z, E_{\nu}\right) .
\end{aligned}
$$

Here, $T_{\text {obs }}$ is the duration of observation, $\mathcal{R}\left(z, E_{\gamma}\right)$ is the comoving rate density and $E_{\gamma}=\alpha_{\gamma \nu} E_{\nu}$ is the radiated electromagnetic energy.

We can then compute the unknown $\alpha_{\gamma \nu}$ factor by equating the expected number of detections from Eqs. 10 or 11 with the expected number of detections from observations.

Note that if we assumed that all sources have the same neutrino luminosity then this step would not be needed, and we could simply compute the overall neutrino flux from the number of detections from observations. However, this step enables the incorporation of the source luminosity distribution, which we can base on the observed $\gamma$ luminosity distributions for the source types in question.

\subsection{Expected total flux at Earth}

To obtain the expected total neutrino flux at Earth for a source type, we integrate over all sources in the universe. We also marginalize over the distribution of 
the expected number of detections from Eq. 9. For continuous sources we obtain

$$
\begin{aligned}
\mathcal{F}_{\nu, \mathrm{S}, \text { cont. }}=\int & d N_{\operatorname{det}} d z d L_{\nu} d \theta \frac{c}{H(z)} \rho\left(z, L_{\nu}, N_{\mathrm{det}}\right) \\
& \times p_{\operatorname{det}}\left(z, L_{\nu}\right) \frac{L_{\nu}}{(1+z)^{2+\alpha}} p\left(N_{\operatorname{det}} \mid \vec{x}\right) .
\end{aligned}
$$

where $\alpha$ is the spectral index of the neutrino spectral density $d N_{\nu} / d \varepsilon_{\nu} \propto \varepsilon^{-\alpha}$, and we expressed $\rho$ as a function of $N_{\text {det }}$ and $L_{\nu}$. This latter takes into account that we do not know the density spectrum of $L_{\nu}$, but can determine it using the density spectrum of $L_{\gamma}$ and $N_{\text {det }}$, and by assuming that $L_{\nu} \propto L_{\gamma}$. We can similarly compute the total flux at Earth for transients:

$$
\begin{aligned}
\mathcal{F}_{\nu, \text { S }, \text { trans. }}=\int & d N_{\operatorname{det}} d z d L_{\nu} d \theta \frac{c}{H(z)} \mathcal{R}\left(z, L_{\nu}, N_{\text {det }}\right) \\
& \times p_{\operatorname{det}}\left(z, L_{\nu}\right) \frac{L_{\nu}}{(1+z)^{3+\alpha}} p\left(N_{\text {det }} \mid \vec{x}\right) .
\end{aligned}
$$

\section{IMPLEMENTATION}

Here we implement the general framework discussed above by considering available information from observations.

\subsection{Source parameter probability densities}

We now turn to $p\left(\vec{x}_{i} \mid B\right)$ and $p\left(\vec{x}_{i} \mid S\right)$.We consider $\vec{x}_{i}=p_{i}$. The probability density of the $p$-value is naturally defined for the background distribution as uniform $\left(p\left(\vec{x}_{i} \mid B\right)=1\right)$. However, the signal distribution $p\left(\vec{x}_{i} \mid z_{i}, L_{\nu, i} S\right)$ cannot be determined without a specific astrophysical signal model and the data analysis framework used in the search. Instead, we adopt a "calibrated" ratio of the background and signal probability densities from Sellke et al. (2001):

$$
\frac{p\left(\vec{x}_{i} \mid B\right)}{p\left(\vec{x}_{i} \mid S\right)}=-e p_{i} \ln \left(p_{i}\right)
$$

for $p_{i}<1 / e$, where $e$ is Euler's number. This ratio is a lower bound over a wide range of realistic $p$-value distributions for the signal hypothesis .

\subsection{Detection probability}

For a given redshift and neutrino luminosity, the probability of detection depends on both the identification of the source through electromagnetic observations and on the detectability of the neutrino signal. Electromagnetic identification determines the completeness $\mathcal{C}\left(z, L_{\gamma}\right)$ of a source catalog, where $L_{\gamma}$ is the electromagnetic luminosity of the source.

\begin{tabular}{llll}
\hline Name & Type & $p$ & Ref. \\
\hline \hline NGC 1068 & AGN & 0.008 & Aartsen et al. (2020a) \\
TXS 0506+056 & blazar & 0.001 & Aartsen et al. (2018a) \\
PKS 1502+106 & blazar & 0.01 & Taboada \& Stein (2019) \\
PKS 1424-41 & blazar & 0.05 & Kadler et al. (2016) \\
AT2019dsg & TDE & 0.002 & Stein et al. (2020) \\
\hline
\end{tabular}

Table 1. Identified astrophysical neutrino sources used in this analysis. $p$ is the $p$-value of the neutrino signal's association with the astrophysical source.

The detectability of the neutrino signal depends on multiple factors, including the neutrino flux and spectrum at Earth, detector sensitivity, and the number density of the source population. In the limit of rare sources, even a single neutrino will be sufficient to identify a source. In this case, detectability will be the probability that a single neutrino is recorded. To obtain this probability for a given source flux, We adopted the expected number of detected neutrinos for a given flux presented by IceCube Collaboration (2016). For a source spectrum $d N_{\nu} / d \varepsilon_{\nu} \propto \varepsilon_{\nu}^{-2.5}$, the flux density corresponding to an expected 1 detected neutrino is $1.2 \times$ $10^{-17} \mathrm{GeV}^{-1} \mathrm{~cm}^{-2} \mathrm{~s}^{-1}\left(\varepsilon_{\nu} / 100 \mathrm{TeV}\right)^{-2.5}\left(T_{\text {obs }} / 1 \mathrm{yr}\right)^{-1}$.

For more common sources, detectability can be approximated with a flux threshold $\mathcal{F}_{\nu, t h}$ such that all those - and only those - sources with flux $\mathcal{F} \geq \mathcal{F}_{\nu, t h}$ are detected. Below we adopt $\mathcal{F}_{\nu, t h}$ from IceCube's 10-year, 90\% confidence-level median sensitivity based on Aartsen et al. (2020a), integrated within $[100 \mathrm{GeV}, 100 \mathrm{PeV}]$. For $E^{-3}$ and $E^{-2}$ spectra this sensitivity is $\mathcal{F}_{\nu, t h, 3}=$ $2 \times 10^{-10} \mathrm{erg} \mathrm{cm}^{-2} \mathrm{~s}^{-1}$ and $\mathcal{F}_{\nu, t h, 2}=10^{-11} \mathrm{erg} \mathrm{cm}^{-2} \mathrm{~s}^{-1}$, respectively. To obtain the sensitivity for an $E^{-2.5}$ spectrum, we consider the geometric mean of $\mathcal{F}_{\nu, t h, 3}$ and $\mathcal{F}_{\nu, t h, 2}$, obtaining $\mathcal{F}_{\nu, t h, 2.5}=2 \times 10^{-11} \mathrm{erg} \mathrm{cm}^{-2} \mathrm{~s}^{-1}$. These values are valid for the northern hemisphere. We account for the fact that IceCube is much more sensitive towards the northern hemisphere by introducing an effective factor of 2 reduction in source completeness.

\subsection{Source types}

Here we introduce the source properties used for the analysis.

\subsubsection{Active galactic nuclei}

One active galactic nucleus (AGN), NGC 1068, has been identified as a likely neutrino source at $2.9 \sigma$ posttrial significance Aartsen et al. (2020a). We use this one detection, with p-value $p=2 \times 10^{-3}$. We adopt the cosmic number density and luminosity function $\rho_{\mathrm{AGN}}\left(z, L_{\gamma}\right)$ for AGNs from the Spitzer mid-infrared 
AGN survey Lacy et al. (2015), with threshold $L_{\gamma} \geq$ $10^{41} \mathrm{erg} \mathrm{s}^{-1}$. We consider AGNs detected as neutrino sources if their neutrino flux is above the threshold $\mathcal{F}_{\nu, t h}$. This flux threshold is adopted based on the measured (90\% C.L. median) sensitivity of IceCube's 10-year search (Aartsen et al. 2020a). We use the typical sensitivity at the northern hemisphere given that IceCube is much more sensitive in this direction, and adopt a factor of 2 reduction in the completeness of the AGN catalog. For a source with neutrino spectral index 2, this corresponds to $\mathcal{F}_{\nu, t h, 2}=6 \times 10^{-13} \mathrm{erg} \mathrm{cm}^{-2} \mathrm{~s}^{-1}$ considering neutrino energies $\varepsilon_{\nu} \in[100 \mathrm{GeV}, 100 \mathrm{PeV}]$. For neutrino spectral index 3 we find $\mathcal{F}_{\nu, t h, 3}=2 \times 10^{-10} \mathrm{erg} \mathrm{cm}^{-2} \mathrm{~s}^{-1}$.

In the detection process, only a few AGNs have been used in neutrino searches, selected based on their $\gamma$-ray brightness (Aartsen et al. 2020a). This limitation ensured that the trial factor in the search remained low. We take this into account by limiting the completeness of our simulated catalog to the 10 brightest sources on the northern hemisphere, i.e. $p_{\text {det }}$ is set to zero for sources with $z$ and $L_{\gamma}$ for which the electromagnetic flux at earth is below a threshold $\left(10^{-9} \mathrm{erg} \mathrm{cm}^{-2} \mathrm{~s}^{-1}\right)$ such that 10 sources are expected.

\subsubsection{Blazars}

For blazars we consider 3 detections (see Table 1). We adopt the cosmic number density and radio luminosity function $\rho_{\text {blazar }}\left(z, L_{\gamma}\right)$ for flat-spectrum radio quasars from Mao et al. (2017), with threshold $L_{\gamma} \geq$ $10^{40} \mathrm{erg} \mathrm{s}^{-1}$. We consider a blazar detected if a single extremely high energy neutrino with energy above $100 \mathrm{TeV}$ is detected from it. For a source with spectral index 2, one extremely high energy neutrino is expected to be detected from a source in a random direction (IceCube Collaboration 2016) for a neutrino flux of $\mathcal{F}_{\nu \text {, single }, 2}=4 \times 10^{-10} \mathrm{erg} \mathrm{cm}^{-2} \mathrm{~s}^{-1}\left(1 \mathrm{yr} / T_{\text {obs }}\right)$ in the neutrino energy range range $\varepsilon_{\nu} \in[100 \mathrm{TeV}, 100 \mathrm{PeV}]$. For spectral index 3 we also have $\mathcal{F}_{\nu, \text { single }, 3}=4 \times$ $10^{-10} \mathrm{erg} \mathrm{cm}^{-2} \mathrm{~s}^{-1}\left(1 \mathrm{yr} / T_{\text {obs }}\right)$.

Similarly to AGNs, we limit the number of blazars in our search catalog. IceCube's 10-year catalog search included about 100 blazars (Aartsen et al. 2020a), we therefore adopt the same number here.

\subsubsection{TDEs}

The detection of one TDE, AT2019dsg, has been reported so far in Stein et al. (2020), which we include in this analysis. We adopt the cosmic rate density of TDEs from Sun et al. (2015), using a minimum TDE luminosity of $10^{44} \mathrm{erg} \mathrm{s}^{-1}$. We treat all TDEs as having identical neutrino luminosity and detectability.

Given the regular electromagnetic follow-up of veryhigh-energy neutrinos released publicly by IceCube, we

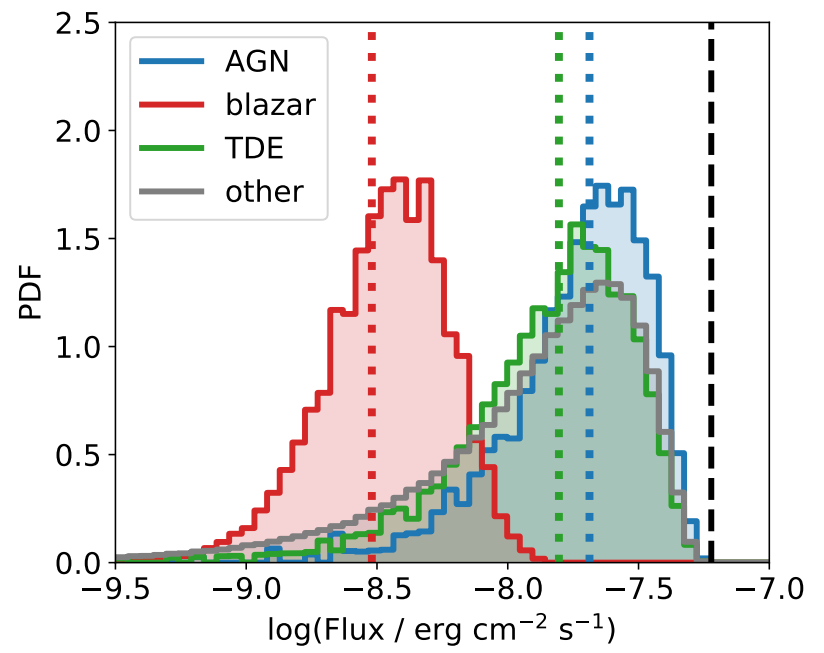

Figure 2. Probability density of total neutrino flux from AGNs, blazars and TDEs based on IceCube's detected sources. For comparison we show the total measured IceCube flux (vertical dashed black line; Aartsen et al. 2020b), and the estimated flux for the three source types using our simple model (Section 3; vertical dotted lines). We assumed an $E^{-2.5}$ astrophysical neutrino spectrum (Aartsen et al. 2020b).

will consider the catalog of TDEs complete out to the distance they can be found through electromagnetic observations. Given the distance range of past identified TDEs (van Velzen et al. 2020), we set their detectable distance at $r_{\max , \mathrm{TDE}}=1 \mathrm{Gpc}$.

We can compute the total fluence needed for the expected detection of a single neutrino analogously for blazars, but using fluence instead of flux. For a source with spectral index 2, we find a neutrino fluence of $\mathcal{S}_{\nu, \text { single }, 2}=0.1 \mathrm{erg} \mathrm{cm}^{-2}$ in the neutrino energy range $\varepsilon_{\nu} \in[100 \mathrm{TeV}, 100 \mathrm{PeV}]$. For spectral index 3 we also have $\mathcal{S}_{\nu, \text { single }, 3}=0.1 \mathrm{erg} \mathrm{cm}^{-2}$.

\section{RESULTS}

We computed the expected cosmic flux for AGNs, blazars, and TDEs using the above prescription. To understand the statistical uncertainty of our results, we obtained the flux probability densities for the three cases based on the probability density $p\left(N_{\text {det }} \mid \vec{x}\right)$ in Eq. 9 . Flux probability densities are computed similarly to Eq. 12 for AGNs and blazars, and Eq. 13 for TDEs, but without marginalization over $N_{\text {det }}$.

The results are shown in Fig. 2. We also list the expected values and $90 \%$ credible intervals in Table 2 . We see that AGNs and TDEs have comparable expected fluxes, while the expected flux from blazars is about a 


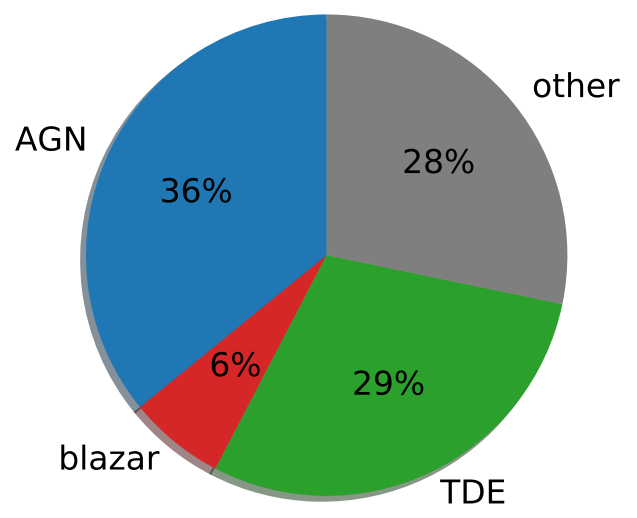

Figure 3. Pie chart of expected fraction of IceCube's neutrino flux from the three source types with directly associated neutrino detections, and the remaining flux from unknown sources (other).

\begin{tabular}{|c|c|c|c|}
\hline \multirow{2}{*}{ Type } & \multicolumn{3}{|c|}{ Flux / $\phi_{\text {IC }}$} \\
\cline { 2 - 4 } & warm-up & simple & full \\
\hline \hline AGN & & 0.34 & $0.36_{-0.27}^{+0.31}$ \\
blazar & 0.1 & 0.05 & $0.06_{-0.04}^{+0.06}$ \\
TDE & 0.55 & 0.26 & $0.32_{-0.24}^{+0.30}$ \\
GRB & & $<0.01$ & \\
CCSN & & $<1.4$ & \\
other & & & $0.28_{-0.25}^{+0.38}$ \\
\hline
\end{tabular}

Table 2. Estimated cosmic neutrino flux as a fraction of IceCube's total measured flux $\left(\phi_{\mathrm{IC}}\right)$. Results are shown for the "warm-up" (Section 2), "simple" (Section 3) and "full" Bayesian (Section 4) models, for AGNs, blazars and TDEs, and the total estimated flux from unknown source types. Error bars indicate $90 \%$ credible interval. For our "simple" model we also show upper limits for GRBs, indicating that non-detection presents are very strict constraint on their allowed contribution to the overall flux due to their very low rate density, and core-collapse supernovae (CCSNe), showing that non-detection does not meaningfully constrain their contribution to the overall neutrino flux due to their high rate density.

factor of 3 lower than these. We also see that, due to the low number of detections so far, the expected flux has considerable uncertainties.

To obtain a pie chart of total fluxes, we look at the properties of the cosmic quasi-diffuse neutrino flux. IceCube detections are consistent with an astrophysical flux following a power-law distribution with spectral index of $2.53 \pm 0.07$ Aartsen et al. (2020b). Therefore, we consider a neutrino spectrum that scales as $E^{-2.5}$ with neutrino energy $E$. Our results would be similar to those presented below if we adopted a somewhat softer spectrum with $E^{-3}$ that best fits the energy distribution of the highest energy neutrinos (Abbasi et al. 2020).

We combine our results together for blazars, AGNs, and TDEs into a pie chart that shows the expected relative abundance of these three source types in the overall flux of high-energy neutrinos IceCube is detecting. The obtained pie chart is shown in Fig. 3.

\subsection{Expected contribution from other source types}

Based on the probability densities of AGNs, blazars and TDEs shown in Fig. 2, we computed the expected contribution from other source types. For this we considered the AGN, blazar, and TDE probability densities to be independent and computed the probability density of their combined neutrino flux, with the boundary condition that the total cannot exceed IceCube's measured total flux. We found that the unknown sources represent at least $10 \%$ (1\%) of IceCube's total flux with $80 \%(98 \%)$ probability.

\section{CONCLUSION}

We computed the expected total high-energy neutrino flux at Earth from AGNs, blazars, and TDEs based on the associations of individual sources with astrophysical neutrinos detected by IceCube, IceCube's sensitivity, and the astrophysical properties and distributions of the three source types. We first carried out a simple derivation of the expected neutrino flux in order to demonstrate how the results scale with the properties of the detections, IceCube and the sources. We then carried out a more detailed derivation that accounts for the statistical uncertainty of the detection process, varying neutrino luminosity within a source type, and the cosmic evolution of source densities and properties. Our conclusions are as follows:

- Despite having detected more blazars with neutrinos than AGNs or TDEs, blazars are expected to be the smallest contributor to the cosmic neutrino flux. We found their contribution to be $3.9_{-2.6}^{+3.7} \times$ $10^{-9} \mathrm{erg} \mathrm{s}^{-1} \mathrm{~cm}^{-2}$ (error bars indicate $90 \%$ credible interval), or a contribution that is $<11 \%$ of the total quasi-diffuse neutrino flux detected by IceCube (at $90 \%$ credible level). This relatively small contribution is due to the fact that blazars are rare, making them much easier to identify through multi-messenger searches than a more common source type with similar total flux contribution.

- AGNs and TDEs represent similar overall contributions. We estimated the AGN flux to be $2.1_{-1.6}^{+1.8} \times$ $10^{-8} \mathrm{erg} \mathrm{s}^{-1} \mathrm{~cm}^{-2}$, while for TDEs the estimated flux is $1.8_{-1.4}^{+1.8} \times 10^{-8} \mathrm{erg} \mathrm{s}^{-1} \mathrm{~cm}^{-2}$. Either AGNs or TDEs could be the majority source of cosmic high-energy 
neutrinos. Their most likely contribution is about $1 / 3^{\text {rd }}$ of the total flux for each.

- One or more, so far unidentified, source types also likely contribute to the overall flux. We estimate their contribution to be $1.7_{-1.5}^{+2.3} \times 10^{-8} \mathrm{erg} \mathrm{s}^{-1} \mathrm{~cm}^{-2}$.

The possibility of unidentified classes of neutrino sources that remained hidden so far makes future observations, and next generation neutrino observatories such as IceCube-Gen2 (The IceCube-Gen2 Collaboration et al. 2020) or KM3NeT (Adrián-Martínez et al. 2016), particularly interesting. The discovery of more AGNs, blazars and TDEs, and the better resolution of the astrophysical high-energy neutrino spectrum, will also be key in enabling the characterization of unidentified source types even if they remain undetected through electromagnetic observations.

The authors thank Christopher Wiebusch for his valuable suggestions. The authors are grateful for the generous support of the University of Florida, DESY, and Columbia University in the City of New York. I.B. acknowledges the support of the National Science Foundation under grant PHY-1911796 and the Alfred P. Sloan Foundation.

\section{REFERENCES}

Aartsen, M., Abbasi, R., Abdou, Y., et al. 2013, Science, 342,1242856

Aartsen, M. G., Ackermann, M., Adams, J., et al. 2018a, Science, 361, eaat1378

—. 2014, Phys. Rev. Lett., 113, 101101

Aartsen, M. G., Abraham, K., Ackermann, M., et al. 2016, ApJ, 833, 3

Aartsen, M. G., Ackermann, M., Adams, J., et al. 2017a, Astroparticle Physics, 92, 30

—. 2017b, ApJ, 843, 112

-. 2018b, Science, 361, 147

—. 2020a, Phys. Rev. Lett., 124, 051103

—. 2020b, arXiv e-prints, arXiv:2001.09520

Abbasi, R., Abdou, Y., Abu-Zayyad, T., et al. 2012, Nature, 484, 351

Abbasi, R., Ackermann, M., Adams, J., et al. 2020, arXiv e-prints, arXiv:2011.03545

Ackermann, M., Ajello, M., Albert, A., et al. 2016, Phys. Rev. Lett., 116, 151105

Adrián-Martínez, S., Ageron, M., Aharonian, F., et al. 2016, Journal of Physics G Nuclear Physics, 43, 084001

Bartos, I., Ahrens, M., Finley, C., \& Márka, S. 2017, Phys. Rev. D, 96, 023003

Blaufuss, E., Kintscher, T., Lu, L., \& Tung, C. F. 2019, in International Cosmic Ray Conference, Vol. 36, 36th International Cosmic Ray Conference (ICRC2019), 1021

Braun, J., Dumm, J., De Palma, F., et al. 2008, Astroparticle Physics, 29, 299

Farr, W. M., Gair, J. R., Mandel, I., \& Cutler, C. 2015, PRD, 91, 023005
Garrappa, S., et al. 2019, Astrophys. J., 880, 880:103

IceCube Collaboration. 2016, GCN, https://gcn.gsfc.nasa. gov/doc/AMON_IceCube_EHE_alerts_Oct31_2016.pdf

Kadler, M., Krauß, F., Mannheim, K., et al. 2016, Nature Physics, 12, 807

Kun, E., Bartos, I., Tjus, J. B., et al. 2021, ApJL, 911, L18

Lacy, M., Ridgway, S. E., Sajina, A., et al. 2015, ApJ, 802, 102

Li, L.-X. 2008, MNRAS, 388, 1487

Mao, P., Urry, C. M., Marchesini, E., et al. 2017, ApJ, 842, 87

Sellke, T., Bayarri, M. J., \& Berger, J. O. 2001, The American Statistician, 55, 62. https://doi.org/10.1198/000313001300339950

Stein, R., van Velzen, S., Kowalski, M., et al. 2020, arXiv e-prints, arXiv:2005.05340

Sun, H., Zhang, B., \& Li, Z. 2015, ApJ, 812, 33

Taboada, I., \& Stein, R. 2019, The Astronomer's Telegram, 12967, 1

Taylor, M., Cinabro, D., Dilday, B., et al. 2014, ApJ, 792, 135

The IceCube-Gen2 Collaboration, :, Aartsen, M. G., et al. 2020, arXiv e-prints, arXiv:2008.04323

van Velzen, S., Gezari, S., Hammerstein, E., et al. 2020, arXiv e-prints, arXiv:2001.01409

Wanderman, D., \& Piran, T. 2010, MNRAS, 406, 1944

Yuan, C., Murase, K., \& Mészáros, P. 2020, ApJ, 890, 25 